\title{
A NOVEL SWARM INTELLIGENCE OPTIMIZED SPECTRUM SENSING APPROACH FOR COGNITIVE RADIO NETWORK
}

\section{C.Jayasri", G.Sakthive}

${ }^{1}$ Research Scholar, Department of Electronics and Instrumentation Engineering, Annamalai University, Chidambaram, Tamilnadu, India, Email-jayasrivijayaraj@gmail.com

${ }^{2}$ Professor, Department of Electronics and Instrumentation Engineering, Annamalai University, Chidambaram, Tamilnadu, India Email-gsauei@gmail.com

Article History:Received:11 november 2020; Accepted: 27 December 2020; Published online: 05 April 2021

Abstract: Spectrum sensing technique have been employed for the detection of various spectrum holes in the transmission of data for the secondary users that do not interfere with the transmission of data of the primary user. The technique known as Cognitive Radio (CR) is the one that efficiently uses the entire spectrum. The primary component of the CR is Spectrum sensing. There are certainly other factors that are considered to be important such as capabilities of cognition and awareness of sensing as well. Identified are different heuristic algorithms that are developed for solving numeric problems in optimization. As the problem has been established as NP-hard, it is essential to bring a low computation complexity heuristic solution. A greedy algorithm is used for optimizing spectrum sharing. Particle Swarm Optimization (PSO) remains an efficient and popular algorithm due to its low need for a tuning parameter, high accuracy, low time for processing, fast convergence, and simplicity. In this work, the PSO has been proposed for spectrum sensing, and this has shown better performance than the Greedy Algorithm used for the CR network spectrum sensing.

Keywords: Spectrum sensing, Cognitive Radio (CR), greedy, and Particle Swarm Optimization (PSO).

\section{INTRODUCTION}

Cognitive Radio Networks (CRN) gains more attention due to its spectrum utilization and resource allocation. The radio access technologies and utilization of spectrum in terms of frequency bands provides wide opportunity to the user to access the resources in cognitive radio network. The unused frequency bands are efficiently identified and utilized in cognitive network increases the spectrum utilization. Moreover, dynamic access to the spectrum is a basic need for the transmitters to adapt to the difference in the quality of channels, congestion of network, and service and interference needs. The CRNs [1] (also known as the secondary networks) will have to coexist along with the ones with legacy (known as primary networks). The primary networks have full rights to access the spectrum without any interference. However, due to this rights to access, the spectrum becomes underutilized. This has resulted in a need to increase the network's capacity to push its research to newer types of exploitation of the wireless medium. The Federal Communications Commission (FCC) defines the regulations and policies for spectrum utilization. The whitespace coalition developed by organizations like Intel, HP, Dell, Google, and Microsoft, explored the possible way to utilize the television band unused spectrum bands. Other cognitive radio networks have been proposed for exploiting opportunistically the "left-overs" and their spectrums using environmental knowledge along with the capability of cognition for adapting their parameters accordingly. The technique of spectrum sensing can enable the network of cognitive radios to reach this goal [2].

Various techniques are evolved in cognitive radio spectrum sensing process for the identification of the PU signal transmission and its presence. The techniques provide a broader utilization of the spectrum for the SUs without any interferences or being intrusive to PUs. Based on this, sensing process is categorized into three types such as Interference based sensing, cooperative sensing, non-cooperative sensing or transmitter detection. Further non cooperative sensing is classified into match filter detection, Euclidean Distance-based Detection, cyclo-stationary feature detection, and energy detection [3]. Among this energy detection is widely used in various research models for spectrum sensing process in cognitive radio networks.

Different heuristic algorithms are being developed to solve problems in numeric optimization. The algorithms may be grouped based on specific criteria which are taken into consideration. They are either based on population, iteration, or stochastic based. Two main groups are seen in algorithms based on which are: The Evolutionary Algorithms (EA) and the Swarm Intelligence-based Algorithms. Also, some metaheuristic techniques such as Simulated Annealing (SA), Genetic Algorithm (GA), TABU Search, Hill Climbing Searches and Greedy Algorithm are evolved to find the optimal solutions. The primary advantage of making use of metaheuristic approaches is that it helps prevent its search space from getting stuck inside the local minima or the local maxima. The techniques help in obtaining better solutions for the application of the network, and for

*Corresponding author: C.Jayasri,

Research Scholar, Department of Electronics and Instrumentation Engineering, Annamalai University, Chidambaram, Tamilnadu, India, Email-jayasrivijayaraj@gmail.com 
this, there can be no practical and optimum solution identified. This is because there is a chance of the global maxima or the global minima not being identified within the stipulated time, and thus most of the network applications become dependent on time for which the process has to be terminated within a specified time. In the case of a CR, the termination criteria will have to be applied mainly for the access of a dynamic spectrum within the CR that has a vital role to play, and in case the decisions are not arrived at in time, the chances of communication may be lost [4].

Optimization refers to the technique that identifies either global maxima or minima for a problem of an objective function, thus avoiding the local maxima or minima [5]. For the problems of computation such as the GA and PSO, there is a need for an optimal solution. However, the local maxima or local minima results into particle loss or computation complexities. There are several other techniques for identifying optimal solutions such as a greedy approach like the GA and the TABU Search. The primary objective of any metaheuristic technique was the prevention of the search space being stuck within the local maxima or local minima [6]. Optimization is the identification of the 'best' solution, which varies for every problem. However, in the case of a statistical problem, this indicates both maximizations, as well as minimization objective function, using different parameters of input.

The PSO is an algorithm of optimization that is population-based in which looking at from the perspective of theory, stopping criteria may not be necessary. At the same time, for the practical problems of optimization, choosing a stopping criterion may be able to influence the rate of convergence and cost of computation to a significant level. All traditional stopping criteria that are used denote error measures that are dependent on known optimum, and this limits the maximum evaluation. Moreover, the maximum evaluation is employed when the global optimum is unknown. Usually, in the PSO, when the global optimum is reached by the particles, the remaining evaluations cannot give rise to any new knowledge and may result in wastage of resources. Through imposing rules to bring the evaluation to an end, the performance of the PSO may be improved. The PSO is an efficient, fast, and simple method of computation which can optimize the problem in an iterative manner attempting to improve the detection of performance and also certain other parameters. The PSO also employs this behavior as swarm intelligence.

For this work, the PSO algorithm to work with CRN spectrum sensing is employed together. The remaining part of this investigation is structured as follows: relevant existing research works are identified and analysed in Section 2. Proposed method is discussed in Section 3 and its experimental results are presented in Section 4. Finally, the observations are concluded in Section 5.

\section{LITERATURE SURVEY}

Shahini and Ansari [7] had proposed to tackle this through decomposing them into two different subproblems. The general issue is of joint resource allocation along with a structure optimization that was formulated in the form of a task of Mixed-Integer Nonlinear Programming that was intractable and NP-hard. It then proposed a scheme of sub-channel allocation for satisfying the rate of Secondary Users (SUs) and their need to remove all constraints of integers. For the next step, it was demonstrated that the overall optimization issue had been condensed as task of convex optimization. Taking into consideration the fractions and their tradeoffs the entire throughput and their need for real-time, as well as non-real-time SUs, were worked on. As the problem of reduced optimization was not observed as optimal solution, Lambert-W function us utilized to bring the optimal solution. Also optimal solutions are obtained by employing Dual Lagrangian decomposition based iterative gradient method. Simulation results had been presented for validating the schemes and their optimality.

Dina Tarek et al., [8] had proposed a new energy-efficient and spectrum efficient scheme to be used for the CR Sensor Networks (CRSNs). They further presented a new architecture for the CRSNs in the IoT where sensor nodes have opportunistic spectrum access and radio frequency based energy harvesting process. After this, a new scheme of energy management was proposed (1) To harvest energy based on the present energy level as energy aware switching (2) To select the cluster head based on the present and past energy levels. This strategy provides an ideal balance between the performance of network and their lifetime. Also, for the intracluster reporting, a strategy of channel management was assigned the best quality channel for both reliability and stability. The results of extensive simulation had demonstrated the effectiveness of this spectrum efficient scheme to show its superiority compared to the other existing schemes.

Wang et al., [9] reported the issues in resource allocation in a multiband multiuser cognitive network through joint spectrum sensing technique. Research model considers the inadequate information of spectrum and identifies the optimized threshold for spectrum sensing along with a strategy of power allocation. This increases the secondary users (SUs) throughout, also reduces the interference to the primary users. As a threefold solution for a mixed-integer non-linear programming (MINLP) optimization problem firstly, the research work presents that the nonconvex MINLP problem has a dimension which is reduced to a significant level. This helps in reformulating the problem of optimization without having to resort to the integer variables. Secondly, there was a demonstration on this which had a simplified formulation that admitted a canonical monotonic optimization along with an E-optimal solution that is achieved by making use of a poly-block outer approximation based algorithm. Thirdly, there was a practical resource allocation and spectrum sensing model 
was introduced to reduce the computation cost. Proposed algorithm and its effectiveness were verified finally through simulations.

Supraja and Pitchai [10] had proposed another new class of heuristics of optimization known as the hybrid optimization. The implementation incorporated two optimization models with similar characteristics. To identify the spectrum patterns using learning algorithms back propagation neural network model is utilized in the research work and it is optimized using hybrid optimization model which is formulated using genetic algorithm and particle swarm optimization. The introduced hybrid optimization model identifies the optimal solution through its optimization characteristics.

Jhajj et al., [11] had made a new proposal for spectrum sensing in cognitive radio networks using Ant Colony Optimization (ACO) - Particle Swarm Optimization (PSO) technique as a hybrid approach. The food foraging characteristics of ants and swarm intelligence are incorporated to obtain optimal results and there by reduces the false alarm probability, error rate and increases the detection probability, throughput.

Songjun Ma et al., [12] had made a new proposal of another novel greedy algorithm for solving problems of wideband spectrum compressive sensing in cognitive radio network. Wideband signals spectrum reconstruction using sub-bands through greedy algorithm was proposed in the research work. This greedy algorithm was able to sense every sub-band range without an apriori information. Simulation results that proved the proposed algorithm had outperformed all existing greedy algorithms like the Orthogonal Matching Pursuit (OMP) that was based on accuracy and speed of computation, Block OMP which was in terms of speed of computation.

Chi Xu et al., [13] had made an investigation in a Heterogeneous Cognitive Radio Network using Superior Selective Reporting (SSR)-based cooperative sensing and Conventional Cooperative Sensing (CCS). The proposed model is an energy harvesting based approach particularly, there are expressions derived for achievable throughput for both these schemes to formulate the problems of nonlinear integer programming to obtain an optimal throughput for the spectrum sensors that sense the channel information of primary user without any interference along with energy harvesting. The observed solutions are obtained based on the cross entropy and compared with greedy and exhaustive search optimization models. Also the discussion incudes the trade-off between CCS and SSR schemes achievable throughput and it was observed that SSR has the ability to outperform the CCS. It was noted that there was an inherent trade-off existing between the channel and time available along with the accuracy of detection. There were numerical results that showed the increase in the spectrum sensors that resulted in the time gains of channels available with a higher priority given to the HCRN in comparison to accuracy of detection.

One of the research areas that is growing fast today is Cooperative sensing, and this is most likely to prove to be an important enabling technology for efficient spectrum sensing. To achieve this spectrum hole white space is need to be identified and to be shared to secondary users without resulting in any interference with the licensed user and his movement. For reliably and swiftly detecting the holes of the spectrum found in the cognitive radios, there is a need to use optimization. Muhammad Rashid Ramzan et al., [4] had made a new study on the various methods of optimization in spectrum searching and its sharing to compare it based on the total error probability for the fading channel.

Alhammadi et al., [14] reported the issues in spectrum mobility when the licensed primary users (PUs) bands are occupied by the Secondary Users (SUs). The spectrum mobility is termed as handoff and it is essential to maintain the handoff delay to minimum to maintain QoS for primary users. To obtain minimum handoff delay, reactive, proactive and hybrid handoff strategies are utilized. Also, to minimize the spectrum handoff service time, Particle Swarm Optimization was incorporated to obtain optimal value for handoff. Compared to other handoff schemes particle swarm optimization attains better performance in minimizing the delay and it was experimentally validated by the experimentation in the research work.

Muthukumaran et al., [15] had made a proposal of another novel and the simple heuristic algorithm for dynamic spectrum access in cognitive network through a scheduled secondary activation link. The algorithm has been presented for a new spectrum underlay wherein both primaries, as well as secondary users, were able to transmit at the same time with cognitive radio networks using the same frequency bands. Firstly, weighted graph is used to model the cognitive radio network. The spectrum sharing issues was reduced by identifying constructed graph sensitive vertex colours. To coordinate secondary transmissions and to obtain best transmission pair, spectrum sharing decision was obtained in spectrum server. Moreover, spectrum servers were used to protect the primary user transmission from interference and it was achieved through suitable power of communication. The gain from proposed algorithms was shown through simulation by extracting the system sum rate from the diversity of transmission selection.

In cognitive radio networks, primary user emulation attack (PUEA) which was a common thread where an intruder duplicates the signal characteristics of Primary User (PU) and project themselves as primary user to mislead the secondary users and to relieve SUs from the spectrum. Ghanem et al., [16], reported the issues due to PUEA in cognitive radio network and using time-difference-of-arrival localization the intruders are detected in the research work. The cost function and localization measurements are solved using Particle Swarm 
Optimization (PSO). The variants of the PSO were developed through changing parameters of the PSO like acceleration constants and inertia weights. The approaches had been presented and then compared to the standard PSO algorithm that was suitable for the problem of localization taken to be an ideal form of guidance to apply algorithms for optimization of wireless positioning techniques. Parameters such as MSE (Mean Square Error) and CDF (Cumulative Distribution Function) are obtained to evaluated the accuracy of the algorithms that were proposed. The results of simulation proved the proposed PSO approaches outperforms in terms of fast convergence and high accuracy compared to conventional methods.

\section{METHODOLOGY}

In linear cooperation framework of spectrum sensing, detection of energy over the linear combination of the nodes is used for decision. For optimal linear detection, it is essential to minimize the false alarm probability and to maximize the probability of detection. Higher probability of detection leads to less interference and higher spectrum efficiency could be achieved when the probability of false alarm is lower. For general systems, the probability distribution function was characterized by Modified Deflection Coefficient (MDC) obtains optimal solutions. Further it was optimized in this research work through a heuristic approach that controls the combining weights. The weight control was achieved using greedy algorithm and optimization is performed using particle swarm optimization.

\subsection{Greedy Algorithm}

The Greedy Algorithm [12] had selected a dictionary vector under applications of compression, pattern recognition, and de-noising. Also, it senses the random sub frequency bands present in the spectrum for data transmission based on the demand of the user. Along with sensing, sub-band recovery also possible in greedy approach which was performed by identifying single peak of the signal in the sub-band. This could be implemented computationally and also very fast.

\section{Pseudocode of Greedy Algorithm [17]}

Greedy $(\mathrm{D}, \mathrm{n})$
$/ / \mathrm{D}$ is a domain and number of solution
For $\mathrm{i}=1$ to $\mathrm{n}$ do
\{
$\mathrm{S}=$ select (D) // selection of solution from D
If (Feasible (Solution, s)) then
Solution = Union (Solution, s)
\}

\section{Merits and demerits of Greedy Algorithm:}

- Identifying solutions may be easy when using a Greedy Algorithm.

- Analyzing run time for the Greedy Algorithms that can be easier compared to other techniques (such as Divide and Conquer).

- The challenging aspect for the Greedy Algorithm needed to work harder for understanding the issues and their correctness. Even when having the right algorithm, it may be challenging to prove if it is right. To prove the Greedy Algorithm as right is an art more than a science.

- At times, the Greedy Algorithms may fail to identify a solution that is globally optimal since they may not consider the entire data. The GA made a choice, and this was dependent on the choices made until now. There was no awareness regarding future choices that may be made.

\subsection{Proposed optimization model}

The proposed particle swarm optimization (PSO) model is a stochastic optimization approach that was based on population designed for mimicking the social behavior of the flock of birds or a school of fish [18]. In the PSO, every potential solution to problems of optimization may be considered as either a bird or fish. The theories had been revealed by utilizing the position (x), velocity (v) and length (D) as vector functions. Where $\mathrm{D}$ refers to the problem variables and its dimensions where the intruder gets confined within the 2D space. Thus it has a value which is equal to 2 . For every such iteration number $(t+1)$, both the position and the velocity of its jth particle obtained from the entire swarm particles (K) had been updated based on equation (1): 


$$
\begin{aligned}
& v_{i}^{t+1}=v_{j}^{t}+c_{1} r_{1}\left(\text { Pbest }_{j}-x_{j}^{t}\right)+c_{2} r_{2}\left(\text { gbest }_{j}-x_{j}^{t}\right) \\
& x_{i}^{t+1}=x_{j}^{t}+v_{i}^{t+1}
\end{aligned}
$$

Wherein $c_{1}$ denotes cognitive coefficients controlling the individual memory and its influence in finding good solutions. $c_{2}$ Denotes a social factor controlling the extent of the motion of a particle that has been influenced using the best solution which was identified by the entire swarm $r_{1}, r_{2}$ were two of the random numbers falling between 0 and 1 that have been used for giving the PSO a randomized ability of search. The variables Pbest and gbest are used to store their best solutions for every particle and the entire swarm. For efficient optimization balancing two crucial factors the global search making the algorithm search for new positons and the current position is fine-tuned using the local search process [16]. Thus, PSO parameters were critical for the problem of optimization to be successful. The flowchart for the proposed PSO is depicted in Figure 1.

In the proposed PSO, the position of a particle represents a combination of weights. The size of the weight vector is equal to the number of CR. On initialization of the PSO, random solutions are generated and the positions are normalized to satisfy the constraint. The PSO algorithm iterates till termination and optimal combination of weights is obtained.

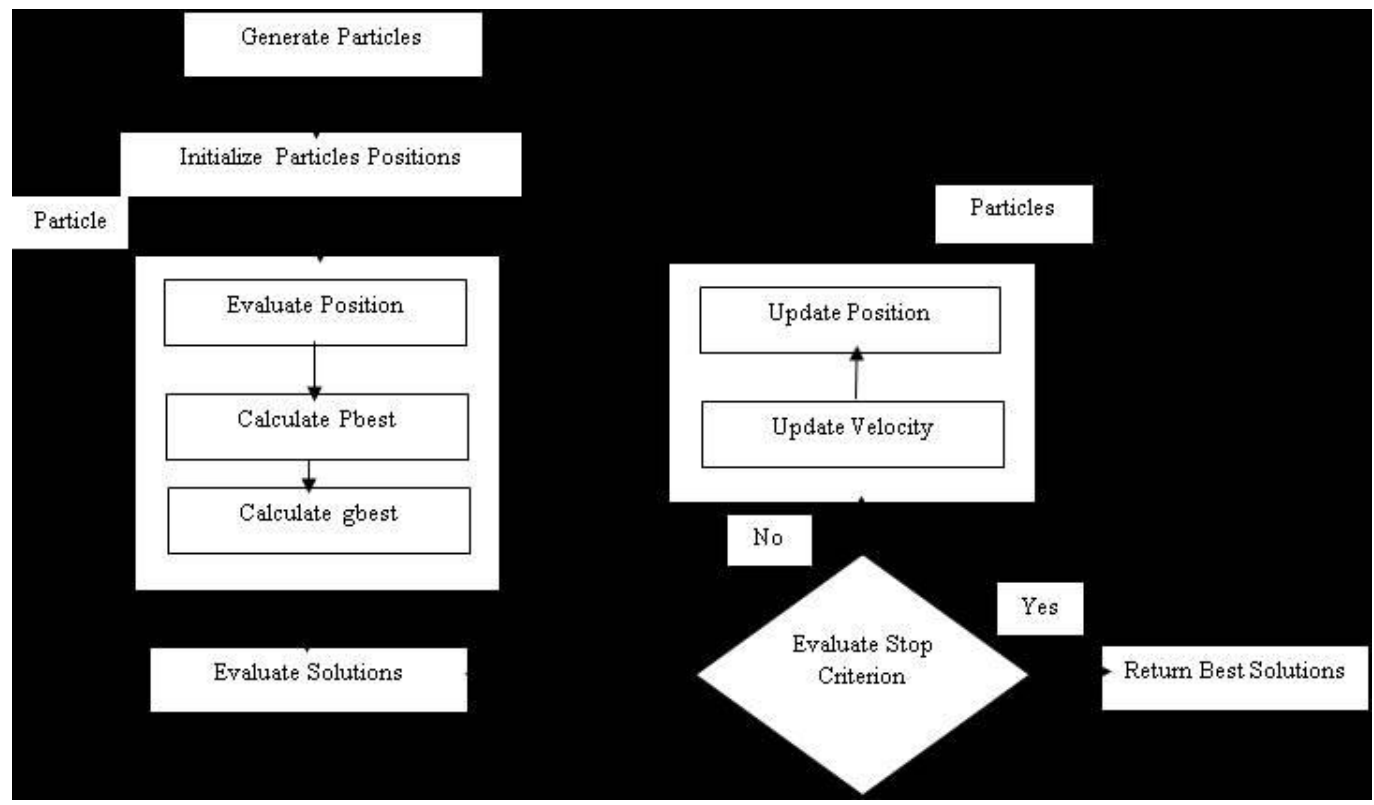

Figure 1 Flowchart for Proposed PSO

The advantages have been summarized below:

- This is easy to be implemented.

- It needs only a few parameters that require adjustments by users.

- There is faster convergence saving the process of spectrum sensing.

- It has high accuracy.

- On being compared to the other heuristics, PSO is affected to a lower extent by the initial solutions.

- It has a less burden in comparison in comparison to the other heuristics.

- There have been different strategies to reduce premature convergence.

\section{RESULTS AND DISCUSSION}

Table 1 shows the parameter of PSO. For experiments, a greedy algorithm and PSO is used. The probability of miss detection and Signal to Noise Ratio (SNR) as shown in tables 2 and 3 and figures 2 and 3 , respectively.

Table 1 Parameters of Particle Swarm Optimization

\begin{tabular}{|c|c|}
\hline Population size & 100 \\
\hline Maximum number of Iteration & 300 \\
\hline
\end{tabular}




\begin{tabular}{|c|c|}
\hline Inertia weight factor & Wmax $=0.9$ and Wmin $=0.4$ \\
\hline Acceleration constant & $\mathrm{C} 1=2$ and $\mathrm{C} 2=2$ \\
\hline Stopping condition & Maximum number of Iteration \\
\hline
\end{tabular}

Table 2 Proposed PSO miss detection probability

\begin{tabular}{|c|c|c|c|}
\hline \multirow{2}{*}{$\begin{array}{c}\text { False alarm } \\
\text { probability }\end{array}$} & \multirow{2}{*}{ Single CR } & \multicolumn{2}{|c|}{ Multiple CR } \\
\cline { 3 - 4 } & 0.96 & Greedy Search & PSO \\
\hline 0 & 0.88 & 0.93 & 0.85 \\
\hline 0.1 & 0.63 & 0.86 & 0.7 \\
\hline 0.2 & 0.54 & 0.61 & 0.34 \\
\hline 0.3 & 0.41 & 0.53 & 0.19 \\
\hline 0.4 & 0.33 & 0.4 & 0.1 \\
\hline 0.5 & 0.2 & 0.32 & 0.09 \\
\hline 0.6 & 0.1 & 0.2 & 0.08 \\
\hline 0.7 & 0.09 & 0.1 & 0.06 \\
\hline 0.8 & 0.09 & 0.09 & 0.06 \\
\hline 0.9 & 0.05 & 0.09 & 0.06 \\
\hline 1 & & 0.05 & 0.05 \\
\hline
\end{tabular}

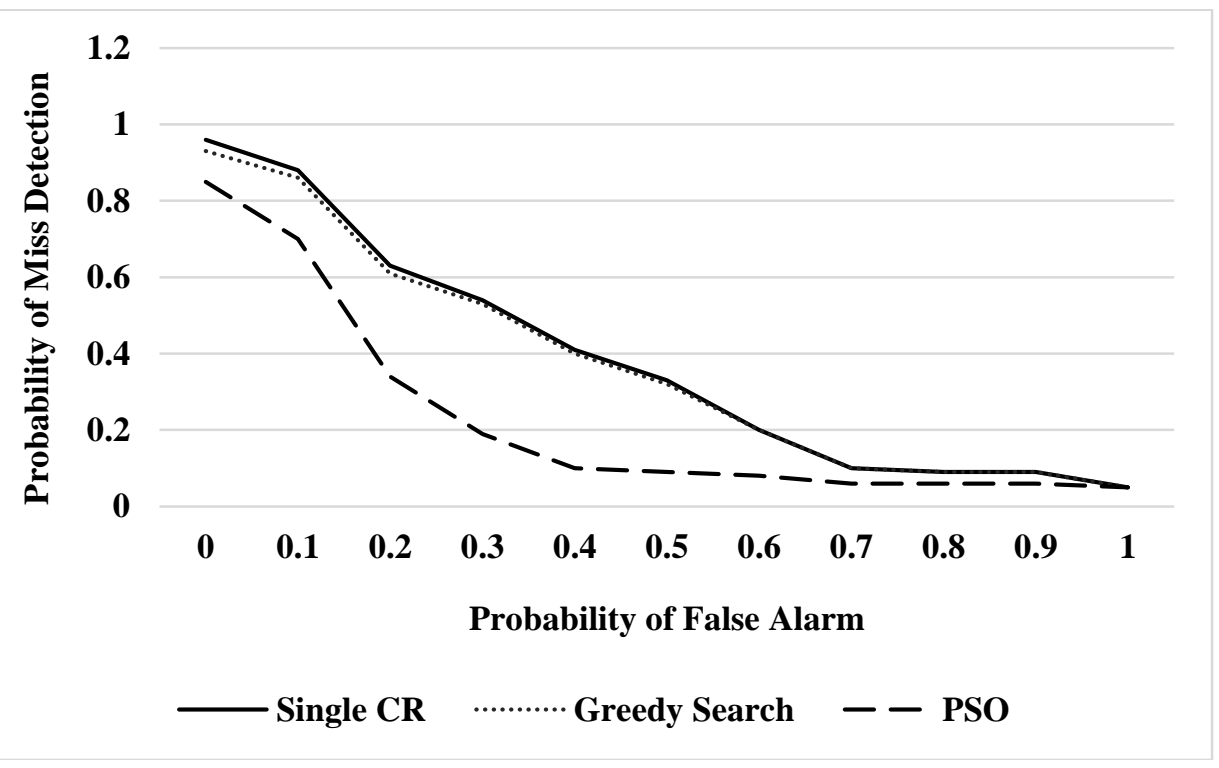

Figure 1 Probability of Miss Detection for Proposed PSO

From the figure 1, it can be observed that the proposed PSO performs better probability of miss detection by $12.15 \%$ and by $8.99 \%$ than Single CR and Greedy Search respectively for the probability of false alarm 0 . The proposed PSO performs better probability of miss detection by $121.57 \%$ and by $120 \%$ than Single CR and Greedy Search respectively for the probability of false alarm 0.4. The proposed PSO performs better probability of miss detection by $40 \%$ and by $40 \%$ than Single CR and Greedy Search respectively for the probability of false alarm 0.8 .

Table 4 Signal to Noise Ratio (SNR) for Proposed PSO

\begin{tabular}{|c|c|}
\hline The technique $(\mathbf{M = 3})$ & SNR in dB \\
\hline Single CR & 7.1 \\
\hline Greedy search & 9.2 \\
\hline PSO & 11.8 \\
\hline
\end{tabular}




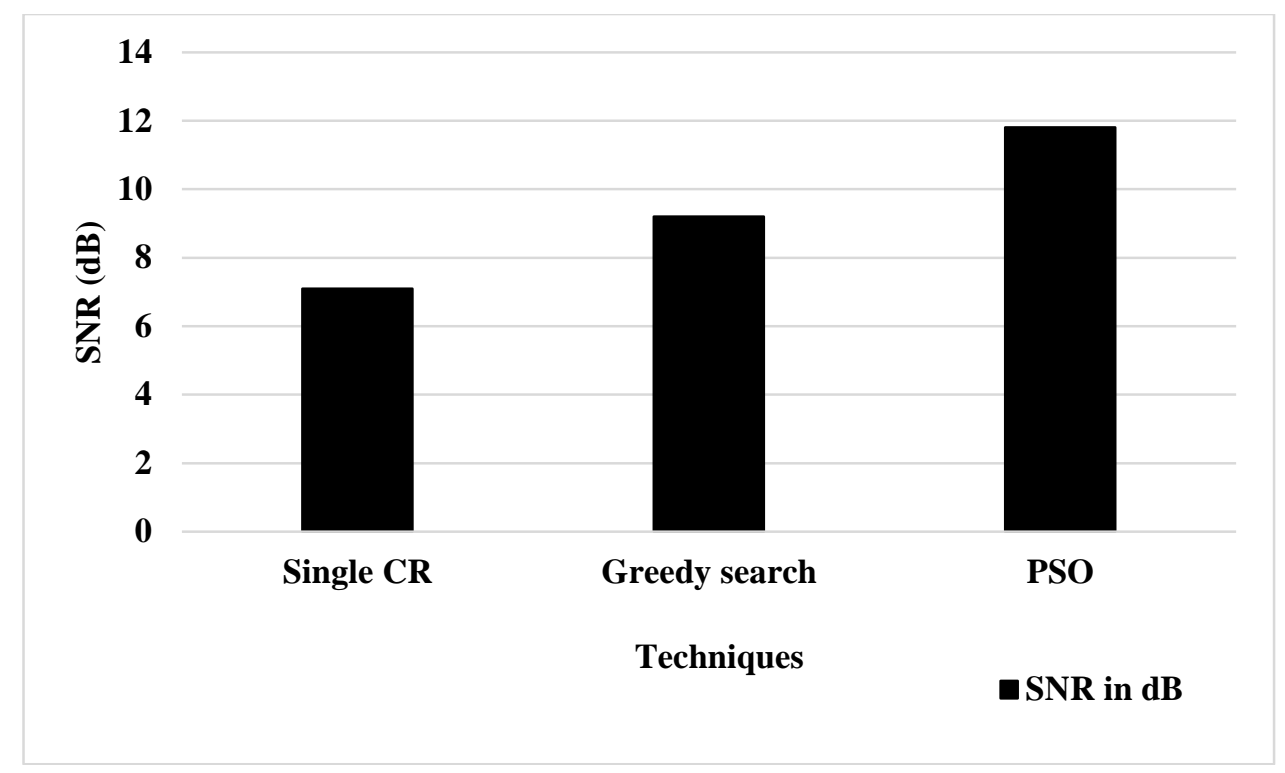

Figure 2 Signal to Noise Ratio (SNR) for Proposed PSO

From the figure 3, it can be observed that the proposed PSO has higher SNR by $49.74 \%$ for single CR and by $24.76 \%$ for Greedy Search respectively.

\section{CONCLUSION}

The Cognitive Radio can access sparse portions of the spectrum and can monitor it for ensuring the Cognitive Radio does not cause any unexpected interference depending on spectrum sensing. For this work, the Greedy Algorithm and the Particle Swarm Optimization had been proposed. The results have proved the PSO to have performed with better probability in the case of misdetection by about $12.15 \%$ and further by about $8.99 \%$ compared to the Single CR and the Greedy Search for the probability of a false alarm of 0. The PSO that has been proposed has performed better by a probability of misdetection which was by about $121.57 \%$ and further by about $120 \%$ compared to the Single CR and the Greedy Search for a probability of false alarm of about 0.4. The PSO that was proposed had performed better in terms of the probability of misdetection by about $40 \%$ and $40 \%$ compared to the Single CR and the Greedy Search for a false alarm probability of about 0.8 . Further, the PSO that was proposed had a higher SNR which was about $49.74 \%$ for the single CR and by about $24.76 \%$ for the Greedy Search.

\section{References:}

Cardoso, L. S., Debbah, M., Lasaulce, S., Mari, K., \& Palicot, J. (2009). Spectrum sensing in cognitive radio networks. Cognitive Radio Networks: Architec-tures, Protocols and Standards.

Yucek, T., \& Arslan, H. (2009). A survey of spectrum sensing algorithms for cognitive radio applications. IEEE communications surveys \& tutorials, 11(1), 116-130.

Mani Shekhar Gupta \& Krishan Kumar (2019). Progression on spectrum sensing for cognitive radio networks: A survey, classification, challenges and future research issues. Journal of Network and Computer Applications, 143, 47-76.

Muhammad Rashid Ramzan, Nadia Nawaz, Alagan Anpalagan (2017). Multi-objective optimization for spectrum sharing in cognitive radio networks: A review. Pervasive and Mobile Computing, 41, 106-131.

Jamal Elhachmi \& Zouhair Guennoun (2016). Cognitive radio spectrum allocation using genetic algorithm. EURASIP Journal on Wireless Communications and Networking. 133, 1-11.

Garg.R, Jhajj.H.k, Saluja.N. (2017).Efficient Spectrum Sensing in Cognitive Radio Networks Using Hybridized Particle Swarm Intelligence and Ant Colony Algorithm. International Journal on Communications Antenna and Propagation (I.Re.C.A.P.), vol. 7(7), pp 586-593.

Shahini, A., \& Ansari, N. (2018). Joint spectrum allocation and energy harvesting optimization in green powered heterogeneous cognitive radio networks. Computer Communications, 127, 36-49.

Dina Tarek, Abderrahim Benslimane, Amira M. Kotb (2020). Survey on spectrum sharing/allocation for cognitive radio networks Internet of Things. Egyptian Informatics Journal, 21(4), 231-239.

Wang, X., Ekin, S., \& Serpedin, E. (2018). Joint spectrum sensing and resource allocation in multi-band-multiuser cognitive radio networks. IEEE Transactions on Communications, 66(8), 3281-3293.

Supraja, P., \& Pitchai, R. (2019). Spectrum prediction in cognitive radio with hybrid optimized neural network. Mobile Networks and Applications, 24(2), 357-364. 
Jhajj, H. K., Garg, R., \& Saluja, N. Implementation of Particle Swarm Optimization Technique for Spectrum Sensing in Cognitive Radio Networks. Vol 7, No 7 (2017)

Songjun Ma, Xiao-Yang Liu, Luoyi Fu, Xiaohua Tian, Xiaoying Gan, Xinbing Wang (2017). On the Greedy Resource Occupancy Threat in Dynamic Spectrum Access. IEEE Transactions on Vehicular Technology, 66(12), 11233-11248.

Chi $\mathrm{Xu}$, Chunhe Song, Haibin Yu (2018). Secure resource allocation for energy harvesting cognitive radio sensor networks without and with cooperative jamming. Computer Networks, 141, 189-198.

Alhammadi, A., Roslee, M., \& Alias, M. Y. (2016, November). Analysis of spectrum handoff schemes in cognitive radio network using particle swarm optimization. In 2016 IEEE 3rd International Symposium on Telecommunication Technologies (ISTT) (pp. 103-107). IEEE.

Muthukumaran.D, Omkumar.S (2019). Heuristic Greedy Method for Spectrum Sensing in Cognitive Radio Network. International Journal of Engineering and Advanced Technology, 9(2), 1454-1459.

Ghanem, W. R., Mohamed, R. E., Shokair, M., \& Dessouky, M. I. (2019). Particle Swarm Optimization Approaches for Primary User Emulation Attack Detection and Localization in Cognitive Radio Networks. arXiv preprint arXiv:1902.01944.

Malik., A, Sharma., A, Vinod., S. (2013) "Greedy Algorithm”, International Journal of Scientific and Research Publications, Volume 3, Issue 8, August 2013.

Kennedy, "Particle swarm optimization," in Encyclopedia of Machine Learning. New York, NY, USA: Springer-Verlag, 2010, pp. 760-766. 